\title{
Os desafios da pesquisa ética com crianças
}

\author{
The challenges of ethical research with children
}

\author{
Rosângela FRANCISCHINI ${ }^{1}$ \\ Natália FERNANDES ${ }^{2}$
}

\begin{abstract}
Resumo
As especificidades da pesquisa com crianças têm ganhado visibilidade nas produções acadêmicas em diversas áreas de conhecimento, com destaque para a Sociologia da Infância, principalmente a partir dos "novos estudos sobre a infância" os quais emergiram ao final da década de 1980. Essa visibilidade acontece devida a questões como: a discussão sobre as imagens da infância, construídas socio-historicamente; o reconhecimento da condição das crianças enquanto atores sociais, portanto, seres competentes, que atribuem significações a suas experiências e contextos; e o reconhecimento das crianças enquanto sujeitos de direitos, reconhecimento este pautado pela Doutrina da Proteção Integral. Permeadas por essas questões, as pesquisas com crianças agregam a questão da Ética nas atividades desenvolvidas na relação pesquisador-criança. No contexto dessa discussão, este trabalho propõe refletir sobre essas questões e os cuidados éticos nesse tipo de pesquisa, considerando a condição da criança enquanto sujeito de direitos. Foi realizado um recorte da produção acadêmica representativa na área da Sociologia da Infância nas duas últimas décadas, identificando quais aspectos aparecem nas preocupações dos autores, quais abordagens norteiam a discussão desses aspectos e, principalmente, que concepção de infância está presente nessa produção.
\end{abstract}

Palavras-chave: Direito da criança; Ética; Pesquisa com crianças.

\begin{abstract}
The specificities of research with children have gained visibility in scientific publications in various areas of knowledge, especially in Sociology of Childhood and the "new studies of childhood", which emerged at the end of the 1980s. This fact is due to discussions about childhood from a socio-historical perspective; the recognition of children as social actors and therefore able to give meaning to their experiences and contexts; and the recognition of children as individuals entitled to rights, according to the Doutrina da Proteção Integral (Full Protection Doctrine). Therefore, research ethics is an essential element of good research governance, especially in terms of activities related to the relationship between the researcher and child. Accordingly, the objective of this study is to examine and explore these issues, considering the child as an individual entitled to rights. A literature review of articles published in the last two decades in the area of Sociology of Childhood was conducted to identify the main issues and topics addressed, the approaches that guided the discussions and, especially, to identify the conception of childhood present in these studies.
\end{abstract}

Keywords: Child right; Ethic; Research with children.

1 Universidade Federal do Rio Grande do Norte, Departamento de Psicologia, Programa de Pós-Graduação em Psicologia. Av. Senador Salgado Filho, s/n., Campus Universitário Lagoa Nova, 59072-970, Natal, RN, Brasil. Correspondência para/Correspondence to: R. FRANCISCHINI. E-mail: <rofrancischini@gmail.com>.

2 Universidade do Minho, Centro de Investigação em Estudos da Criança, Departamento de Ciências Sociais de Educação. Braga, Portugal. 
A partir do final da década de 1980 e, principalmente, na década de 1990, assistiu-se à emergência e consolidação da Sociologia da Infância, uma área de estudos a qual tem, dentre seus pressupostos fundantes, o reconhecimento da criança enquanto "ator social" (A. James \& James, 2004; James, Jenks, \& Prout, 1998; James \& Prout, 1997; Prout \& James, 1990; Sirota, 1998a, 1998b). Paralelamente, deu-se, em nível internacional, a promulgação, em 1989, da United Nations Convention on the Right of the Child (UNCRC, Convenção das Nações Unidas sobre os Direitos das Crianças) que reconhece a condição de "sujeitos de direitos" para essa parcela da população.

A convergência desses dois fatores - sujeitos de direito e atores sociais -, observada, inclusive, na produção em Sociologia da Infância (Freeman, 1998; Mayall, 2000), trouxe implicações para os pesquisadores, dentre as quais destaca-se a defesa da participação ativa das crianças em pesquisas que Ihe dizem respeito. Fernandes (2009, p.87) diz com relação a isso que "...ao desafiar as tradicionais concepções, a Sociologia da Infância é o mais poderoso aliado do movimento de direitos das crianças; ao defender para ela um conjunto de direitos políticos sustenta a possibilidade de concretização da sua ação e participação". Em acréscimo, e mesmo decorrente dessa mudança epistemológica, comparecem, também, nas produções acadêmicas relacionadas à área, propostas de mudanças metodológicas a partir do questionamento sobre as (im)possibilidades e limitações dos métodos e procedimentos (principalmente entrevistas e questionários fechados) empregados nas pesquisas com adultos.

Nesse sentido, a literatura tem enfatizado a adoção de métodos participativos, centrados no interesse das crianças (Lange \& Mierendorff, 2009; McNamee \& Seymour, 2012; O'Kane, 2000). A esse respeito, Prout e James (1990) têm sinalizado a adequação da etnografia, por reconhecer e possibilitar a participação da criança na construção dos dados da pesquisa. Recursos visuais, recorrentes na abordagem etnográfica, principalmente fotografias, têm demonstrado possibilitar às crianças uma maior participação na pesquisa e a focalização, no duplo sen- tido (o da lente e o da escolha do objeto/acontecimento a ser captado), naquilo que the parece significante (Clark, 2010; Thomas \& O'Kane, 1998; Vakaoti, 2009).

A opção pela Etnografia, certamente, não é a única. Métodos participativos, conforme sinalizado acima, tem possibilitado ao pesquisador uma aproximação do universo dos sujeitos e um desenho da pesquisa que, se não apaga a assimetria das relações entre investigador-investigado, ao menos minimiza o caráter de poder que frequentemente a caracteriza.

Esse reconhecimento colocou os pesquisadores diante de questões e desafios que, até então, pouco ou quase nada apareciam nas pesquisas. Dizem respeito às responsabilidades éticas do pesquisador quando do manejo dessa população (Alderson, 1995; Christensen \& Prout, 2002; Morrow \& Richards, 1996; Thomas \& O'Kane, 1998).

Assim, as questões éticas "tradicionais" que permeiam os processos de investigação com seres humanos adultos estendem-se às investigações com crianças e adolescentes, não obstante as particularidades das quais se revestem, acrescidas, ainda, de questões emergentes. Nesse sentido, dentre as principais questões éticas em pesquisa, cabe ao investigador assegurar às crianças investigadas:

- O direito à explicitação da proposta da pesquisa, com ênfase nos seus objetivos, métodos e propósitos, garantindo todas as informações necessárias para que o Termo de Consentimento Livre e Esclarecido (TCLE) seja efetivamente uma escolha/ opção desses sujeitos e/ou daqueles que por eles são responsáveis;

- O respeito aos direitos de privacidade e confidencialidade, nas condições de construção e de divulgação dos dados;

- As informações sobre os possíveis benefícios da pesquisa e a garantia de que o mesmo não será submetido a situações consideradas de risco;

- O direito de não-participação em situações que julgar inapropriadas, inadequadas ou que the causem algum incômodo, e o direito de deixar de participar da investigação em qualquer momento de seu percurso; 
- O direito a ter acesso aos resultados da investigação, antes mesmo de se tornarem públicos;

- A garantia de que os custos da participação na pesquisa (por exemplo: deslocamento, alimentação, materiais, etc.) serão de responsabilidade do investigador e que a participação deverá se dar em horários que não prejudiquem outras atividades exercidas pelos sujeitos, incluindo as remuneradas. Trata-se, nesse caso, de crianças e adolescentes em situação de trabalho, nos vários contextos em que exercem atividades as quais, apesar de proibidas pela legislação, ainda persistem. São exemplos, no Brasil, crianças trabalhando nas ruas, nas feiras, nas olarias, na construção civil, em atividades na agricultura, dentre outras. Inclui, também, adolescentes em condição de aprendiz prevista na legislação (Brasil, 1990).

Considerando esse conjunto de salvaguardas, nos caminhos éticos da investigação com crianças, a importância que assumem para respeitar sua imagem como sujeito humano com direitos, continua, no entanto, a subsistir nos diversos contextos de investigação. Esses direitos são, muitas vezes, ignorados, desvalorizados ou, ainda, mal interpretados.

Alderson (1995) e Alderson e Morrow (2004, 2011), ao defenderem que se considere nas questões éticas de investigação com crianças uma trilogia entre direitos, deveres e danos/benefícios, assinalam alguns princípios orientadores sobre essa questão que auxiliam sua compreensão. O trabalho do investigador é de tessitura dessas três dimensões de forma que resultem do processo de pesquisa dinâmicas que contribuam para fortalecer os direitos, acautelar os deveres e salvaguardar o bem-estar das crianças. No que diz respeito à dimensão dos deveres, Alderson (1995) defende que esta é uma questão essencialmente deontológica, uma vez que exige que haja um questionamento, por um lado, da equidade nas relações que se estabelecem, quer entre crianças e adultos, quer entre crianças e seus pares e, por outro, da adequabilidade dos objetivos e dos métodos utilizados na investigação.

No que diz respeito à dimensão dos direitos, chama-se especial atenção para a cautela que o pesquisador deve ter durante o processo de inves- tigação com relação à interferência ou intrusão nos mundos de vida das crianças. Deve estar especialmente atento à salvaguarda da liberdade e da vontade de participação da criança na investigação, e ainda à proteção contra dinâmicas que possam atentar contra direitos básicos de proteção e provisão. Finalmente, no que diz respeito à dimensão dos danos e/ou benefícios, Alderson (1995) chama especial atenção para uma dimensão mais praxeológica da investigação, no sentido de mobilizar estratégias de redução ou prevenção dos danos e aumentar a possibilidade de usufruir de benefícios a partir da investigação.

É possível concluir a partir disso que o investigador deve promover dinâmicas de pesquisa que façam uma gestão equilibrada entre os interesses da criança, dos pais, dos profissionais e da sociedade em geral, considerando, dessa maneira, a complexidade de que se reveste a vida social nas múltiplas redes que a compõem e por entre as quais a infância vai acontecendo. Não basta um olhar isolado sobre a criança para salvaguardar eticamente o processo de pesquisa, uma vez que ela, enquanto ator social, estabelece relações com uma multiplicidade de outros atores que inevitavelmente colocam-se nesse processo.

Essas questões não esgotam, certamente, a amplitude dos aspectos éticos envolvidos na atividade de pesquisa. Além disso, são questões não resolvidas, algumas inclusive bastante polêmicas, que necessitam, ainda, maior debate e diálogo entre os pesquisadores, as próprias crianças e os adultos que delas se ocupam. O recorte que a seguir se apresenta, ilustra alguns contornos da visibilidade dessas questões nas pesquisas com crianças e nas reflexões teóricas produzidas a partir da Sociologia da Infância.

\section{Método}

Recorte de um projeto mais amplo de Pós-doutorado em Estudos da Criança - Sociologia da Infância -, este artigo, ao propor recorrer à produção acadêmica representativa na área nas duas últimas décadas, tem, por fonte de dados, as publicações referidas na International Society of Sociology (ISA) 
e na European Society of Sociology (ESA). Foram incluídas produções em língua inglesa e francesa, devido à escolha por periódicos divulgados por essas duas associações de Sociologia. Assim, a produção em português não foi contemplada.

No site de ambas as associações foram identificadas as publicações que apresentavam em seus resumos (abstracts) a expressão "Sociology of Childhood". Os períodos compreendidos foram de março de 1986 a dezembro de 2012 e de janeiro de 1980 a dezembro de 2012, disponibilizados, respectivamente, pela ISA e pela ESA. Foram identificados, na ISA, 20 artigos e, na ESA, 22. As publicações que configuram apresentações e/ou comentários de livros foram excluídas. Com relação às preocupações dos autores com questões relativas à ética na pesquisa com crianças, observou-se que elas aparecem, como objeto de reflexão, em seis dos 42 artigos lidos.

Portanto, o corpus desta pesquisa é composto por esses seis textos, sendo três deles teóricos (Moran-Ellis, 2010; Sirota, 2010; Van Krieken, 2010) e outros três com pesquisa de campo (Huggins \& Rodrigues, 2004; Newman, MacDougall, \& Baum, 2009; Vakaoti, 2009), muito embora, seja estabelecido diálogo com os demais artigos mais adiante. A leitura dos mesmos foi realizada tendo por orientação um roteiro elaborado previamente, onde foram registrados, para cada artigo, vários aspectos que neles compareciam, dentre os quais, um resumo, observações da pesquisadora e questões específicas sobre ética, de interesse imediato para o recorte que aqui se propõe.

\section{Resultados e Discussão}

Iniciando pela questão mais ampla, qual seja, a noção de infância que permeia as reflexões dos autores, e que, portanto, reflete nas preocupações éticas, todos situam suas investigações no campo da Sociologia da Infância, condição essa decorrente da escolha para a construção do corpus desta investigação. Essa condição, no entanto, não implica que tenha sido observada unanimidade entre eles no que se refere à eleição dos pressupostos dessa área de conhecimento.
Assim, prevalece o pressuposto "criança enquanto ator social", presente em quatro artigos (Huggins \& Rodrigues, 2004; Moran-Ellis, 2010; Van Krieken, 2010; Vakaoti, 2009), mas aparecem, ainda, a ênfase nos Direitos das crianças (Sirota, 2010; Van Krieken, 2010) e o reconhecimento de sua capacidade de expressar-se e produzir interpretações sobre a realidade, seus objetos e acontecimentos. A respeito desse último aspecto, dois dos artigos (Moran-Ellis, 2010; Newman et al., 2009) abordam, enfática e prioritariamente, a perspectiva de dar voz às crianças, ouvi-las não somente no sentido acima referido, de produção de interpretações sobre a realidade, mas, também, no que se relaciona aos aspectos próprios da investigação, como, por exemplo, seus métodos e a elaboração da agenda para a construção dos dados. Defendem, portanto, uma noção de infância pautada na capacidade das crianças, nem sempre considerada pelos adultos, de expressarem-se e no direito de serem escutadas nas questões que the dizem respeito.

No que se relaciona especificamente às preocupações dos autores com questões relativas à ética na pesquisa com crianças, conforme pôde ser observado, elas aparecem, enquanto objeto de reflexão, em seis dos 42 artigos analisados, sendo eles, portanto, os que estão aqui sendo considerados. No entanto, dentre os demais artigos, alguns autores afirmam terem obtido o TCLE dos participantes ou de seus responsáveis (Farrell, Tayler, \& Tennent, 2012) e/ou que seguiram as determinações dos Códigos de Ética (profissionais) ou dos Comitês de Ética (vários). Outros, ainda, indicam referências bibliográficas de investigações que têm esse aspecto contemplado (Bühler-Niederberger, 2010; Tisdalla, Kay, \& Punch, 2012).

Considerando-se a ética na pesquisa com crianças e adolescentes, conforme observado anteriormente, essa discussão está diretamente relacionada aos aspectos eleitos pelos teóricos na configuração da noção de infância. Acrescenta-se, ainda, que nos artigos com trabalho de campo a escolha dos participantes também tem papel determinante no desenho da pesquisa e nas demais escolhas do investigador. Assim, como há dois textos que têm, 
como sujeitos participantes, crianças e adolescentes em situação de rua, são abordados temas específicos relacionados a essa condição. Como exemplo, podemos citar as representações que são produzidas sobre essa população e os cuidados do investigador para a entrada em campo.

Em pesquisa anterior de orientação etnográfica com jovens em situação de rua (Matias \& Francischini, 2010) são discutidos esses dois aspectos. Assim, a revisão da literatura tornou explícita a representação predominantemente negativa que é construída em torno dessa população, incluindo uma homogeneização indevida e inadequada no que diz respeito às características e condições dessa vivência. Em relação ao processo de entrada em campo com grupo de pessoas em situação de rua, destaca-se a complexidade desse processo e os mecanismos de negociação necessários: muitos deslocamentos e flexibilidade do pesquisador para que possa responder adequadamente às várias e diversificadas demandas de formas de participação e envolvimento junto ao grupo. Assim, um dos principais desafios à pesquisa etnográfica com essa população foi a necessidade constante de negociações de sentido e a abertura para novas possibilidades de invenção técnica e experiência ética.

Retomando, então, os aspectos éticos presentes nos artigos aqui considerados, estes serão apresentados em dois blocos: o primeiro, onde foram incluídos os artigos com pesquisa de campo e, o seguinte, caracterizado por trabalhos teóricos. Iniciando pelas pesquisas com trabalho de campo, prevalece a abordagem sobre o TCLE e as questões a ele relacionadas, como, por exemplo, a participação da criança, uma vez que o Termo de Consentimento é justamente a expressão da concordância do sujeito para participar da pesquisa. Em estudos com crianças e adolescentes, portanto, de 0 a 17 anos, no Brasil (Brasil, 1990), o TCLE é solicitado aos pais ou cuidadores. Até que ponto ele reflete a concordância da criança ou adolescente é uma questão que merece reflexão.

Com relação a essa questão, em um dos trabalhos analisados (Newman et al., 2009) os autores afirmam que na Austrália, nos procedimentos de investigação com as crianças, é obrigatória a presen- ça do adulto. Mais que o consentimento, o responsável pela criança permanece no locus da investigação. Os autores observam a necessidade de se criar espaços onde as crianças possam expressar-se sem a presença do adulto, visto que isso pode resultar em restrições nas ações e discursos.

Ainda sobre o Termo de Consentimento, em um dos trabalhos, o pesquisador solicitou-o aos participantes a cada procedimento empregado na pesquisa. Segundo o autor, essa opção é eticamente a mais adequada pois permite aos sujeitos maior controle em todo o percurso da investigação.

Em outro trabalho (Huggins \& Rodrigues, 2004), igualmente com crianças em situação de rua, o problema colocado pelos investigadores foi: a quem deveriam pedir permissão/consentimento para a participação das crianças na pesquisa na impossibilidade de recorrer aos responsáveis por elas? Na ausência dos pais ou cuidadores desses sujeitos, optaram por solicitar o consentimento aos mais velhos do grupo. Permanece, no entanto, enquanto desafio a ser abordado, a questão da escolha/opção, por parte das crianças e adolescentes, de participarem ou não da investigação.

Uma outra questão ética abordada, ainda considerando os artigos com trabalho de campo, diz respeito à postura do investigador. O tema mais recorrente, presente, inclusive, na produção acadêmica para além dos artigos aqui em discussão, diz respeito às diferenças de poder entre pesquisador (adulto) e pesquisado (criança) (Grover, 2004; Morrow, 2008; Punch, 2002a, 2002b). Como opção para minimizar essa condição, o autor (Vakaoti, 2009) propõe que o investigador assuma uma postura de abertura - reflexividade -, adotando, assim, a possibilidade de alterações/adaptações nos métodos em função da realidade e dinâmica do campo. O mesmo autor discute, ainda, enquanto questão ética, os métodos de investigação, apontando a importância da etnografia e dos métodos participativos, com ênfase para a observação participante. Além dessas questões, Vakaoti (2009) faz referência, também, à possibilidade de que os sujeitos tenham alguma forma de compensação financeira por estarem participando da pesquisa. 
A esse respeito, Huggins e Rodrigues (2004) afirmam terem oferecido uma "compensação" (lanche ou dinheiro) para as crianças que participaram da investigação. Não há, no entanto, qualquer observação avaliativa sobre esse procedimento por parte dos autores. Justificam-no, todavia, no sentido de compensarem o dinheiro que os participantes (supostamente) deixariam de ganhar ao participarem da entrevista, uma vez que estariam impedidos de trabalhar. Essa é uma questão delicada, ausente, entretanto, nas discussões sobre a ética na pesquisa com crianças. Dentre outros aspectos, pode resultar em implicações quanto à livre e consentida escolha dos participantes e, igualmente, nas relações de poder entre pesquisador e pesquisado. Merece, portanto, cuidado e atenção específicos.

Considerando, agora, os três artigos teóricos, observou-se, inicialmente, que têm a especificidade de tratarem da questão da ética na pesquisa com crianças no interior da discussão sobre a Sociologia da Infância em seus respectivos países. Assim, Austrália, França e Reino Unido são os contextos discutidos, com acento nas especificidades dessa área de conhecimento, principalmente no que diz respeito aos temas abordados, decorrentes das condições históricas e culturais dessas sociedades em geral e da criança, em particular. Entretanto, as três abordagens acentuam a importância do discurso dos Direitos da Criança e as implicações teórico-metodológicas na pesquisa com esses sujeitos. A partir dessas observações gerais seguem suas particularidades.

Iniciando pela Austrália, Van Krieken (2010) afirma que a discussão sobre ética na pesquisa com crianças tem o domínio da medicina. Portanto, os problemas e métodos abordados são aqueles típicos da pesquisa em saúde em geral e em medicina. Afirma, ainda, que a Sociologia ocupa uma "posição marginal" "na definição da agenda dos Estudos da Criança" (p.241). Afirma, ainda, que houve crescimento da importância dos Comitês de Ética naquele país.

A propósito dos Comitês de Ética e ingressando, agora, no domínio da Sociologia da Infância na França, Sirota (2010) afirma que a sujeição dos pesquisadores, principalmente daqueles de orienta- acaba resultando em prejuízos para a pesquisa. Observa, ainda, a ausência, nas pesquisas com crianças do país, de debates sobre as questões éticas.

No Reino Unido, Moran-Ellis (2010) observa que a questão ética na pesquisa com crianças "não é uma arena sem problemas". A autora circunscreve essa questão no contexto de discussão, nas estruturas de governo, da proteção dos interesses dos sujeitos pesquisados e da intenção de salvaguardá-los de práticas não éticas. Observa, no entanto, que o efeito pode ser, também, de controle da pesquisa, uma vez que determinados temas e questões julgados importantes pelo investigador podem não ter aprovação por parte daqueles que trabalham nessas estruturas governamentais. Além disso, observa que a "pesquisa convencional com crianças" nesse país, tem sido palco de transgressões éticas e aponta os trabalhos de Alderson (1995) e Alderson e Morrow (2004) como referências importantes nessa questão.

Os questionamentos referentes aos métodos de pesquisa têm destaque nas reflexões do autor e são abordados em termos de preferência por abordagens consideradas "mais éticas" (as participativas), as quais possibilitam amenizar o poder do pesquisador nas relações com os sujeitos pesquisados. Afirma, no entanto, que o debate permanece em aberto e que há, ainda, várias questões que precisam de continuidade.

\section{Considerações Finais}

A recente história dos "novos estudos sobre a criança" justifica, em parte, o que se pôde constatar com este estudo: a quase ausência de discussões sobre a questão da ética na pesquisa com esses sujeitos, a emergência de novos desafios para o pesquisador e o estágio embrionário atrelado ao enfrentamento ainda tímido desses desafios. No entanto, nas pesquisas aqui abordadas, onde identificou-se o aparecimento desse tema, pode-se afirmar sua importância.

Ressalta-se, ainda, que nesta pesquisa o corpus é constituído por publicações que têm, em seu resumo, a expressão "Sociologia da Infância". A discussão sobre ética na pesquisa com crianças, embora com predominância nessa área do conhe- 
cimento, não está circunscrita apenas a ela; na última década, tem aparecido de forma crescente, como tema em produções acadêmicas de modo geral, revelando a necessidade e a importância de ser colocada em pauta (Cruz, 2008; Kramer, 2002; Vasconcellos \& Sarmento, 2007).

Retomando a Sociologia da Infância, a partir de uma concepção desse período da vida pautada pelo reconhecimento das crianças enquanto atores sociais, seres competentes, os quais participam ativamente nos diversos contextos em que estão inseridos, construindo significações sobre os objetos, eventos e acontecimentos de seu cotidiano e que têm o direito de serem ouvidos nos assuntos que Ihes dizem respeito, os autores afirmam e assumem a necessidade de uma mudança metodológica. Enfatizam a opção por métodos de pesquisa que possibilitem, e mesmo promovam, a participação das crianças investigadas.

Impõe-se, então, o primeiro desafio: a exigência do TCLE. Pode-se considerar esse aspecto a partir de duas dimensões: numa dimensão burocrático-formal é inquestionável que o consentimento informado na pesquisa com crianças está indelevelmente ligado à tutela adulta. Assim sendo, a concordância formal de participação na pesquisa, atestada pelo referido TCLE, é assumida pelos pais ou responsáveis legais da criança. Do ponto de vista da legislação, a competência da criança não é, portanto, reconhecida; isso depende do atingimento da maioridade, a qual dá-se, no Brasil, quando o sujeito completa 18 anos, o mesmo ocorrendo em Portugal.

Uma outra dimensão, a partir da qual pode-se encarar esse consentimento, tem a ver com a autoria da criança no processo. Se em muitos contextos esse aspecto não tem qualquer importância, desde que se assegure o que consta na primeira dimensão, há outros contextos para os quais são mobilizadas imagens distintas de crianças e infância, onde se reconhece que estas têm também uma voz e uma identidade a serem respeitadas nos procedimentos de pesquisa, onde é, portanto, fundamental considerar também o seu consentimento. A esse propósito, nos textos analisados, é interessante perceber a diversidade que se encerra no pequeno número de trabalhos destacados para análise, diversidade essa que ilustra aquela que é, indiscutivelmente, uma das caraterísticas mais presentes na categoria geracional infância: a diversidade.

Assim, em determinados contextos da Austrália, para além do consentimento, é exigida a presença do adulto durante os procedimentos da pesquisa, como forma de assegurar que a criança esteja protegida. Conforme James e James (2009), o desafio, então, para os pesquisadores da infância, é fazer a distinção entre idade e competência e garantir a correta avaliação dessa última. As bases sobre as quais as competências das crianças foram tradicionalmente avaliadas, sobretudo aquelas pautadas por determinadas vertentes predominantes em Psicologia do Desenvolvimento no decorrer do século XX, necessitam de revisão; idade, ou mesmo condição biológica, isoladamente, não se sustentam como critérios para atestar a (suposta) incompetência das crianças, como as pesquisas tem demonstrado.

Os avanços recentes em Psicologia do Desenvolvimento, decorrentes, sobretudo, do acesso aos teóricos da vertente denominada Sócio-histórica (por vezes, sociocultural ou, ainda, histórico-cultural), representada, principalmente, por Vygotsky, têm possibilitado uma revisão dos conceitos e práticas em pesquisa nessa área do conhecimento. "Aprendemos, com esse autor, que 'é a sociedade e não a natureza que deve figurar em primeiro lugar como fator determinante do comportamento do homem" " (Vygotsky, 1995, p.31). Assim, a constituição do ser humano, das funções psicológicas superiores, está diretamente relacionada às formas de vida e de interação, às práticas sociais e, mais especificamente, discursivas, características dos diversos momentos da história social" (Francischini \& Campos, 2008, p.103). Há, portanto, uma possibilidade de diálogo entre essas duas áreas do conhecimento, na perspectiva de estudos interdisciplinares sobre a infância.

Retomando a Sociologia da Infância e as questões éticas na pesquisa com crianças, as quais aparecem nos artigos aqui analisados, um segundo desafio se impõe: a necessidade de se manejar, dentre a diversidade de processos que tecem as relações pesquisador/pesquisado, a assimetria nessas relações, caracterizada, quase sempre, por 
relações de poder do adulto sobre a criança, com imposição do primeiro e apagamento do segundo. Essa prática, que permeia as relações adulto-criança em nossa sociedade, decorre das diferentes posições e papéis atribuídos e/ou assumidos social e culturalmente, nos diversos contextos e instituições frequentados pelos sujeitos.

Assim, as soluções apontadas pelos autores, conforme identificado nas pesquisas, dizem respeito à adoção de métodos e procedimentos participativos. De acordo com Bakhtin (1992a, 1992b), o funcionamento interindividual, e portanto, as relações que se estabelecem entre investigador e investigado, é de natureza semiótica e dialógica, isto é, mediado por signos e práticas sociais do sujeito com o outro. É nesse jogo dialógico/dialético que se constitui o sujeito, sua consciência.

Nesse sentido, a atitude de silenciar ou a de possibilitar a expressão da voz da criança é, antes de tudo, uma questão ética fundamental; as interações que se estabelecem no percurso da investigação podem tanto favorecer quanto impedir o exercício da responsabilidade, da autonomia e mesmo de expansão dos recursos que a criança mobiliza em sua compreensão da realidade.

Além disso, ouvir o que as crianças têm a dizer sobre suas experiências, consultá-las a respeito das questões que afetam seu dia a dia, na família, na escola e nos demais contextos de socialização, possibilita, ao pesquisador, e àqueles implicados em programas de intervenção junto a essa população, ter acesso a um universo de significações próprio, o qual pode subsidiar, inclusive, as propostas de intervenção a elas dirigidas. O reconhecimento dessa voz, estende-se, ainda, à possibilidade de participação, como cidadãos, em tomadas de decisão de aspectos que Ihe dizem respeito. Conforme observou-se a partir das leituras da produção anteriormente referida neste artigo, essa possibilidade permanece, ainda, em construção.

Por fim, uma nota sobre os Comitês de Ética. Os trabalhos aqui em pauta sinalizam que esses comitês podem, por vezes, dificultar, e mesmo impedir, o desenvolvimento da pesquisa. Não é objetivo deste trabalho discutir tudo o que está implicado com relação a isso. Eles existem, acredita-se, gados não tenham seus direitos violados. No entanto, é importante observar que é recorrente, entre os investigadores, o apontamento de problemas enfrentados ao submeter seus projetos a esses comitês, o que sinaliza para a necessidade de um maior diálogo entre esses atores.

Os apontamentos deste trabalho representam, de alguma forma, as principais questões e desafios que os novos estudos da infância suscitaram. Ainda em processos de discussão, essas questões necessitam de maior debate e diálogo entre as diversas áreas de conhecimento que vêm se ocupando de compreender a infância, as crianças, seus anseios e suas possibilidades enquanto atores nos diversos contextos que frequentam.

"Precisamente por causa de sua complexidade, um entendimento compreensivo da infância não pode ser realizado/alcançado através de uma perspectiva epistemológica e disciplinar única: um fenômeno complexo requer estudo interdisciplinar, assim, o estudo da infância tem que ser compreendido como uma atividade multi e interdisciplinar" (A. James \& James, 2009).

\section{Colaboradores}

Todos os autores contribuíram na concepção e desenho do estudo, análise de dados e redação final do artigo.

\section{Referências}

Alderson, P. (1995). Listening to children: Children, ethics and social research. London: Barnardo's.

Alderson, P., \& Morrow, V. (2004). Ethics, social research and consulting with children and young people. London: Barnardo's.

Alderson, P., \& Morrow, V. (2011). The ethics of research with children and young people: A practical handbook. London: Sage Publications.

Bakhtin, M. M. (1992a). Estética da criação verbal. São Paulo: Martins Fontes.

Bakhtin, M. M. (1992b). Marxismo e filosofia da linguagem. São Paulo: Hucitec. (Originalmente publicado em 1929).

Brasil. Presidência da República. (1990). Lei Federal $n^{\circ}$ 8.069/90, de 13 de julho de 1990. Dispõe sobre o Estatuto da Criança e do Adolescente e dá outras providências. Brasília: Autor. Recuperado em agosto 19, 2013, de http://www.planalto.gov.br/ccivil/LEIS/ L8069.htm 
Bühler-Niederberger, D. (2010). Childhood sociology in ten countries: Current outcomes and future directions. Current Sociology, 58(2), 369-384.

Christensen, P., \& Prout, A. (2002). Working with ethical symmetry in social research with children. Childhood, 9(4), 477-497.

Clark, A. (2010). Young children as protagonists and the role of participatory, visual methods in engaging multiple perspectives. American Journal of Community Psychology, 46(1-2), 115-123. http://dx.doi.org/10. 1007/s10464-010-9332-y

Cruz, S. H. V. (Org.). (2008). A criança fala: a escuta de crianças em pesquisas. São Paulo: Cortez.

Farrell, A., Tayler, C., \& Tennent, L. (2012). Building social capital in early childhood education and care: An Australian study. British Educational Research Journal, 30(5), 622-632.

Fernandes, N. (2009). Infância, direitos e participação: representações, práticas e poderes. Porto: Edições Afrontamento.

Francischini, R., \& Campos, H. R. (2008). Crianças e infâncias, sujeitos de investigação: bases teórico-metodológicas. In S. H. V. Cruz (Org.), A criança fala: a escuta de crianças em pesquisa (pp.102-117). São Paulo: Cortez.

Freeman, M. (1998). The sociology of childhood and children's rights. The International Journal of Children's Rights, 6(4), 433-444.

Grover, S. (2004). Why won't they listen to us? On giving power and voice to children participating in social research. Childhood, 11(1), 81-93.

Huggins, M. K., \& Rodrigues, S. (2004). Kids working on paulista avenue. Childhood, 11(4), 495-514.

James, A., \& James, A. (2004). Constructing childhood: Theory, policy and practice. Basingstoke: Palgrave Macmillan.

James, A., \& James, A. (2009). Key concepts in childhood studies. London: Sage.

James, A., Jenks, C., \& Prout, A. (1998). Theorising childhood. Cambridge: Polity Press.

James, A., \& Prout, A. (Eds.). (1997). Constructing and reconstructing childhood: Contemporary issues in the sociological study of childhood. London: Falmer Press.

Kramer, S. (2002). Autoria e autorização: questões éticas na pesquisa com crianças. Cadernos de Pesquisa, 116, 41-59.

Lange, A., \& Mierendorff, J. (2009). Method and methodology in childhood research. In J. Qvortrup, W. Corsaro, \& M. Honig (Eds.), The palgrave handbook of childhood studies (pp.78-95). Basingstoke: Palgrave Macmillan.

Matias, H. J. D., \& Francischini, R. (2010). Desafios da etnografia com jovens em situação de rua: a entrada em campo. Psicologia: Reflexão e Crítica, 23(2), 243-252.

McNamee, S., \& Seymour, J. (2012). Towards a sociology of 10-12 year olds? Emerging methodological issues in the 'new' social studies of childhood. Childhood, 20(2), 156-168.
Mayall, B. (2000). The sociology of childhood in relation to children's rights. The International Journal of Children's Rights, 8(3), 243-259.

Moran-Ellis, J. (2010). Reflections on the sociology of childhood in the UK. Current Sociology, 58(2), 186-205.

Morrow, V., \& Richards, M. (1996). The ethics of social research with children: An overview. Children \& Society, 10(2), 90-105.

Morrow, V. (2008). Ethical dilemmas in research with children and young people about their social environments. Children's Geographies, 6(1), 49-61.

Newman, L. A., MacDougall, C. J. M., \& Baum, F. E. (2009). Australian children's accounts of the closure of a car factory: Global restructuring and local impacts. Community, Work \& Family, 12(2), 143-158.

O'Kane, C. (2000). The development of participatory techniques: Facilitating children's views about decisions which affect them. In P. Christensen \& A. James (Eds.), Research with children: Perspectives and practices. London: Falmer Press.

Prout, A., \& James, A. (1990). A new paradigm for the sociology of childhood? Provenance, promise and problems. In A. James \& A. Prout (Eds.), Constructing and reconstructing childhood. London: Falmer Press.

Punch, S. (2002a). Interviewing strategies with young people: The 'secret box', stimulus material and taskbased activities. Children and Society, 16(1), 45-56.

Punch, S., (2002b). Research with children: The same or different from research with adults? Childhood, 9(3), 321-341.

Sirota, R. (1998a). 'L'Emergence d'une sociologie de I'enfance: évolution de l'objet, évolution du regard'. Éducation et Sociétés 2 (Special issue), 9-34.

Sirota, R. (Ed.). (1998b). Éducation et Sociétés 2 and 3. Lyon: ENS Éditions. (Special issue: Sociologie de l'enfance).

Sirota, R. (2010). French childhood sociology: An unusual, minor topic or well-defined field? Current Sociology, 58(2), 250-271.

Tisdalla, E., Kay, M., \& Punch, S. (2012). Not so 'new'? Looking critically at childhood studies. Children's Geographies, 10(3), 249-264.

Thomas, N., \& O'Kane, C. (1998). The ethics of participartory research with children. Children and Society, 12(5), 336-348.

Vakaoti, P. (2009). Researching street-frequenting young people in Suva: Ethical considerations and their impacts. Children's Geographies, 7(4), 435-450.

Van Krieken, R. (2010). Childhood in Australian sociology and society. Current Sociology, 58(2), 232-249.

Vasconcellos, V. M. R., \& Sarmento, M. J. (Orgs.) (2007). Infância (in)visível. Rio de Janeiro: Junqueira \& Marin .

Vygotsky, L. S. (1995). Obras escogidas. Madri: Visor Distribuciones.

Recebido: setembro 27, 2013

Versão final: junho 9, 2014

Aprovado: outubro 6, 2014 
